\title{
What Causally Insensitivite Events Tell us About Overdetermination
}

\section{Sara Bernstein}

Billy and Suzy each throw a rock through a window; the rocks hit the window at precisely the same time; the window shatters. Either rock-throw would have been sufficient for the shattering of the window. This is a paradigmatic case of causal overdetermination, in which there are multiple causes sufficient to bring about the same effect.

However, holding that overdetermination ever really occurs faces the challenge of distinguishing it from joint causation: if only Suzy had thrown the rock, the shattering would have been different: the window would have shattered with slightly less force, for example. ${ }^{1}$ Multiple causes collaborate to bring about an effect, and the absence of one of the causes would have resulted in a slightly different effect. Does every purported case of causal overdetermination turn out to be a case of joint causation underdescribed ${ }^{2}$

No. In this paper, I isolate a distinctive kind of causal overdetermination in which the effect is not significantly changed by an extra cause, and I explain why it undergirds the distinction between joint causation and overdetermination. I argue for the following thesis: some overdetermined effects are physically insensitive in ways that jointly caused, non-overdetermined effects aren't, and these cases aren't reducible to joint causation. I call these overdetermination cases insensitivity cases. Insensitivity cases are central to the distinction between joint causation and overdetermination, the modal profile of events, and our understanding of redundant causation more generally.

Roadmap: In section 1, I propose and defend a thesis about the metaphysics of certain overdetermined effects. I do not give an analysis of all causal overdetermination; rather, I identify what makes a certain kind of overdetermination case distinctive, and what distinguishes this type of case from joint causation. In section 2, I apply this machinery to Schaffer's “trumping preemption." I suggest that, when fully described, trumping preemption is a case of insensitive overdetermination, and thus Lewis' "influence" account of counterfactual causation is less equipped to handle this

\footnotetext{
${ }^{1}$ Those who hold that overdetermination is prevalent include Schaffer (2003), Sider (2003), and Paul

${ }^{2}$ For an early example of this reasoning, see Bunzl (1979).
} 
counterexample than previously thought.

\subsection{Causal Overdetermination}

Returning to the case of Billy and Suzy (hereafter: Two Rocks), we have a case of redundant causation insofar as both $c 1$ (Suzy's throwing of the rock) and $c 2$ (Billy's throwing of the rock) are sufficient for e (the shattering of the window) to occur. The major species of redundant causation are overdetermination and preemption. It is easy to see that Two Rocks is not a case of preemption, due to the symmetry between $\mathrm{c} 1$ and $\mathrm{c} 2$.

In cases of preemption, one cause preempts the other from bringing about the effect. This can happen either when the preempting cause somehow interrupts or deters the preempted causal process from running to completion (early preemption) or when it brings about the effect before the preempted cause can do so (late preemption). A early preemption variant of Two Rocks is a case in which Suzy throws her rock before Billy is going to throw his, and Billy, disheartened, decides not to throw at all. A variant of Two Rocks that would be a case of late preemption is one in which Billy and Suzy throw at the same time, but Suzy's rock is faster, and shatters the window before Billy's rock can come into contact with it. The original Two Rocks case, on the other hand, cannot fall under the heading of preemption, because $\mathrm{c} 1$ and $\mathrm{c} 2$ bring about the shattering of the window at exactly the same time.

One can concede that Two Rocks isn't a case of preemption but deny that it is overdetermination by suggesting that it isn't genuinely a case of redundant causation, but is instead a case of joint causation. All parties can agree that a variant of Two Rocks in which the window does not shatter from the force of one rock alone is a case of joint causation. However, some maintain that even the original Two Rocks case is not overdetermination, but is rather joint causation.

The natural strategy for one who denies that Two Rocks is genuine overdetermination is to argue that the window shattering via Suzy and Billy is a different effect from the window shattering due to one rock. Perhaps the window shattering via Suzy and Billy has a property-say, shattering with extra force-sufficient to render the effect a case of joint causation. After all, it takes both rock-throws to cause the shattering in precisely the way that it occurs. 
Such a view is called fragility. According to Lewis, we can call an event fragile if "it could not have occurred at a different time, or in a different manner." ${ }^{3}$ The implication is that taking events to be modally fragile renders all causal overdetermination joint causation underdescribed: at a maximally specific level of description, every case of overdetermination is actually a case of joint causation.

A similar move takes events to be fine-grained. This strategy involves acceptance of a very fine level of granularity at which we "slice" events, generating a great many events. The most prominent proponent of this strategy is $\mathrm{Kim}^{4}$, who holds that events are individuals that instantiate a property at a time. There is an event for every <object, property, time $>$ triple such that $\mathrm{o}$ instantiates $\mathrm{p}$ at $\mathrm{t}$; only fine grained events enter into causal relationships. Events differ when they differ in their constituents, properties, or times. The window shattering forcefully is a distinct event from the mere window shattering. On this view, there are countless events located in the same place and time. Arguably, one could use fine-granularity to argue against overdetermination in a similar way that modal fragility does; for example, it takes both Billy's rock and Suzy's rock to bring about the exact group of <object, property, time> triples that they do-the window would not have shattered with quite so much force and in the precise way it did unless both rocks had been thrown. ${ }^{5}$ Here I focus mostly on modal fragility, but I take my points to generalize to fine-grained events as well.

Let's call the causal relata-refinement strategy JOINT CAUSATION (for short: JOINT). JOINT captures the idea that Two Rocks is actually a case of joint causation because the shattering of the window via both rocks is a different shattering than the one that would have occurred with one rock. JOINT effectively eliminates the Two Rocks sort of overdetermination, since each cause makes a difference to the way the effect occurs, thus turning every putative case of overdetermination into a case of joint causation. In section 1.2, I will argue against JOINT. But JOINT hones in on what is puzzling about Two Rocks: it's difficult to think of the case as overdetermination when

\footnotetext{
${ }^{3}$ See Lewis (1986b)

${ }^{4}$ See Kim $(1976)$

${ }^{5}$ There is at least one ordered triple that is straightforwardly overdetermined: the window shattering, coarsely described. For example, both Billy's and Suzy's rocks are individually sufficient to cause the occurrence of triple <window, shattering, 2:30pm>. But Kimian events are essentially fine-grained, and no two rocks will cause exactly the same group of ordered triples that one rock would cause. Thanks to an astute referee for this point.
} 
the shattering is so different from the one that would have occurred had only one rock been thrown.

One could hold that one difference between the insensitive alarm sounding due to one rock and the insensitive alarm sounding due to two rocks is that the two differ in their relational properties: the former has the property being caused by one rock and the latter has the property being caused by two rocks. But this move subtly changes the subject away from the central challenge of the Bunzl-style objection to overdetermination. That objection (upon which JOINT is based) requires intrinsic physical differences between the singly-caused and overdetermined outcomes. Distinguishing between the outcomes based on their causal origins does not directly address the Bunzl-style objection because causal origins are neither intrinsic nor physically instantiated (as in the case of, for example, the extra force of an overdetermined shattering).

\subsection{Causally Insensitive Effects}

Let's temporarily grant JOINT. What would convince us that not every case of putative overdetermination is susceptible to JOINT? We need a case where $c 1$ and $c 2$ are each sufficient to bring about $e$ in precisely the way it occurs-that is, make precisely the causal contribution necessary to bring about $e$-while together making no more than the causal contribution necessary to bring about $e$ had only one cause occurred. Here is such a case, modified from Two Rocks:

(Window Alarm) A window is rigged to an alarm. The alarm sounds if the window is shattered. Billy and Suzy throw their rocks; each hits the window at precisely the same time. The alarm sounds. ${ }^{6}$

Billy and Suzy overdetermine the sounding of the alarm. Here there is no temptation to apply JOINT to the sounding of the alarm, because the alarm doesn't sound differently whether one or two rocks shatters the window. ${ }^{7}$ What is the difference between Two Rocks and Window Alarm?

\footnotetext{
${ }^{6}$ The sounding of the alarm is an acoustic event that depends on a particular arrangement of particles.

7 There might be minute differences in the movement of particles between the alarm sounding due to one rock and the alarm sounding due to two rocks. I do not take these microscopic physical differences to be significant. See below for more on significant differences.
} 
In Window Alarm, the effect doesn't reflect the force with which it is caused: the alarm is insensitive with respect to the force of both causes. In contrast, the shattering of the window is highly sensitive with respect to the additive force of both causes. We relegate Two Rocks to the category of joint causation because the way the window shatters is highly sensitive to the force with which it is caused: each cause must occur for the window to shatter in precisely the way it does. But the way that an alarm sounds isn't sensitive to the number of causes: the sounding is precisely the same no matter how many rocks shatter the window. Now consider the following contrast case to Window Alarm:

(Multi-Volume Window Alarm) There is a window rigged to an alarm with many volumes. Wanting to know how many vandals are breaking her windows, the neighbor programs the alarm to sound with volume correlative to the force with which the window is broken. Billy and Suzy throw their rocks; each hits the window at precisely the same time. The alarm sounds loudly.

In this case, Billy and Suzy jointly cause the sounding of the alarm. The distinctive feature of Multi-Volume Window Alarm is that the sounding of the alarm is sensitive to the number of causes: how it occurs varies with the number of causes.

From this pair of cases, we can extract the following thesis: in some cases of overdetermination, the effect is insensitive with respect to the amplifying force of multiple causes. Having an extra cause or causes does not significantly alter the way that the effect occurs. An effect is sensitive, in contrast, if the manner in which it occurs is dependent on the number of causes. The lesson can be summed up in the slogan: in cases where it's unclear whether the effect is overdetermined or jointly caused due to acceptance of modal fragility, sensitivity of the effect distinguishes joint causation from overdetermination. ${ }^{8}$ Even if JOINT is a successful strategy for responding to some purported cases of overdetermination, insensitivity cases are not easily undermined by the JOINT maneuvers: multiple causes are individually sufficient to bring about $e$ in

\footnotetext{
${ }^{8}$ There are many different kinds of overdetermination, including overdetermination of effects by mental and physical properties implied by nonreductive physicalism, to which this analysis might not apply. Nonreductive physicalists generally hold that mental properties do not contribute physical force or additive energy to a mentally caused outcome. In "Overdetermination Underdetermined", I argue that this commitment yields an unsatisfactory picture of mental causation. Either the nonreductive physicalist must give an account of why mentally caused outcomes are insensitive effects, or give an account of the exact causal contribution of the mental cause. This argument generalizes to overdetermination of the macro level by the micro level (as in Merricks, 2007).
} 
precisely the way it occurs, while together making no more than the causal contribution necessary to bring about $e$ had only one cause occurred.

Why should whether or not a case is one of overdetermination depend on properties of the effect? Several reasons. First: the effect provides the necessary information for distinguishing between joint causation and overdetermination in cases where it is ambiguous. Consider the following diagram, in which circles represent causal relata ${ }^{9}$ at particular times and arrows represent causation:

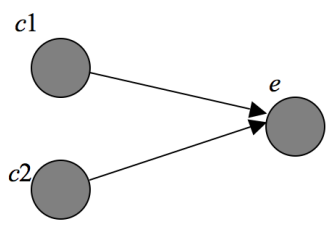

Diagram 1: Joint Causation or Overdetermination?

Suppose that we do not antecedently know whether $\mathrm{c} 1$ and $\mathrm{c} 2$ represent partial or full causes of $e$ : all we know is that $\mathrm{c} 1$ and $\mathrm{c} 2$ occur simultaneously and each make some sort of causal contribution to $e$. Then this diagram is insufficient for distinguishing between joint causation and overdetermination, for in both cases, there are multiple causes with complete causal processes that hit the effect at once. Suppose that all we have is the diagram, and we want to figure out if $e$ is overdetermined or jointly caused.

The normal procedure for classifying a case of redundant causation involves looking to see which processes "run to completion". ${ }^{10}$ Consider the following case:

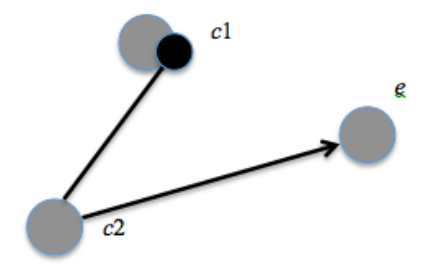

Diagram 2: Early Preemption

In this diagram, one process fails to "run to completion," making it a case of early preemption. And in a case of late preemption, though both causal processes run to completion, one cause brings about the effect before the other's impact:

\footnotetext{
${ }^{9}$ I remain neutral on what the causal relata are. They could be property instances, facts, or events.

${ }^{10}$ It is unclear exactly what it is for a causal process to "run to completion". For discussions of various notions of this, see Bernstein (2014b), Hall and Paul (2013) and Dowe (2000).
} 


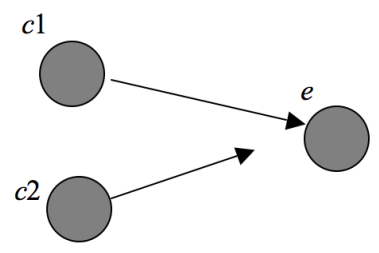

Diagram 3: Late Preemption

But in Diagram 1, the causal processes leading up to the effect do not furnish the information we need, for both joint causation and overdetermination involve two causal processes running to completion, each impacting the effect at the same time. With just the causal elements antecedent to the effect, the causal diagnosis of the diagram is underdetermined.

We need to know whether the effect is sensitive or insensitive in order to deem Diagram 1 joint causation or overdetermination. If we don't know the nature of the effect, we don't know how to categorize the case. If the effect is sensitive, the diagram represents joint causation. If the effect is insensitive, the diagram represents overdetermination. ${ }^{11}$

The lesson generalizes beyond neuron diagrams. Note how, in order to turn Window Alarm into a case of joint causation, all we have to do is replace the insensitive alarm with the sensitive alarm. Consider these diagrams of Window Alarm and MultiVolume Window Alarm:

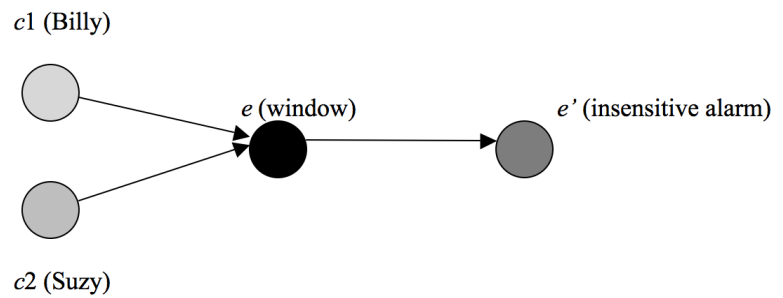

Insensitive Alarm

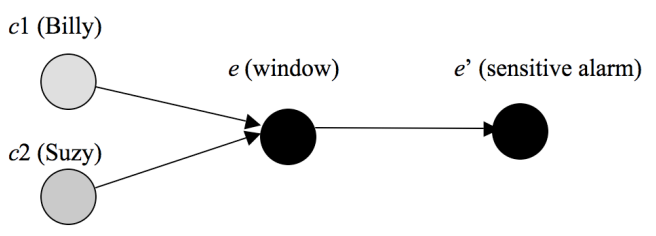

Sensitive Alarm

Blackened neurons represent sensitive effects; grey neurons represent insensitive

\footnotetext{
11 Note that one need not hold that neuron diagrams capture all of the relevant facts of every case of causation in order to take this claim on board. A neuron diagram captures the time at which each cause occurs, the completion of each causal process, and the occurrence of the outcome-most of the relevant information relating to the distinction between joint causation and overdetermination. Neuron diagrams capture causal contribution, but not distinctions between partial and full causal contributors. This missing piece - whether each cause is partial or full-is determined in relation to the sensitivity of the effect.
} 
effects. ${ }^{12}$ In each case, multiple causes bring about an initial sensitive effect (the shattering of the window). But the difference lies solely in the two alarms. The causal structures are identical except for their effects, which categorize each causal structure as overdetermination or joint causation.

\subsection{Physical and Counterfactual Insensitivity}

Exactly what is insensitivity? Distinguish between physical insensitivity, in which an actual event is impervious to extra physical force, ${ }^{13}$ and counterfactual insensitivity, in which an actual event fails to vary counterfactually with other events. Either sort of insensitivity may be had in relation to a number of causes or in relation to the force that those causes exert. Physical sensitivity tracks any physical change in an outcome; for example, particles moving differently and being located in different places than they would have been had the causes been different. Counterfactual sensitivity tracks whether the same event would have occurred across possible worlds.

Physical insensitivity is a property an actual effect has in relation to a class of its actual causes. More formally:

[Physical Insensitivity] An effect $e$ is physically insensitive to a class of causes $C$ if counterfactual variations in what subset of $C$ is instantiated don't make significant physical differences to $e .^{14}$

For example: in Window Alarm, call the class of rock-throws $C$ and call each rock throw $c 1, c 2, c 3$, and so on. The event of the alarm sounding is insensitive with respect to which and how many rock throws occur. There might be minute physical differences between the alarm sounding due to one rock and the alarm sounding due to two: for example,

\footnotetext{
${ }^{12}$ For simplicity, I am examining only one dimension of sensitivity. For example, one could have an alarm that changes volume based on the number of points of contact with the window, and pitch based on the force of shattering.

${ }^{13}$ Though I focus on physical forces traditionally construed, I take "force" to cover any dimension of a cause that varies. This notion is not strictly limited to pure "push and pull." For example, differing doses of a drug may be considered causally differential, and a body might be insensitive to differential doses, but a dose is not, strictly, a force.

${ }^{14}$ Whether an event is insensitive is relative to background conditions and laws. Thanks to an astute referee for drawing attention to this condition.
} 
there will be ever so slight differences in the gravitational forces of the alarm, leading to minute acoustic differences in the noise it emits. But there will be no significant physical differences to the sounding of the alarm.

I fully admit that the line between significant physical differences and insignificant differences is arbitrary. But this arbitrariness does not threaten the idea that some events are more physically impervious to change than others, and that events on the far end of the imperviousness spectrum can be called "insensitive". It is undeniable, for example, that there are more physical differences between the singly-caused and doubly-caused window-shatterings than there are between the single-caused and doubly-caused alarmsoundings.

Why is insensitivity held "in relation to a class of causes"? Because the sounding of the alarm isn't insensitive with respect to everything. Consider the following modification of Window Alarm:

(Well-Equipped Delinquent) There is a window rigged to an alarm. Billy is poised to throw his rock. Suzy, having grown tired of the inefficiency of rock-throwing, has procured a missile launcher. Billy throws his rock at the window, and Suzy launches her missile.

Here's what does not happen in this case: the window shatters, and the alarm sounds. It is more likely that the entire window, the alarm, and the entire house to which they are attached explode with massive force. The sounding of the alarm is physically insensitive to rock throws, but sensitive to missiles. The lesson: physical insensitivity is a property of an effect held in relation to certain types of causes, but not all of them.

Often effects are not physically insensitive simpliciter, but rather insensitive with respect to thresholds of force. Call an effect strongly insensitive if no addition or subtraction of causes past the minimally sufficient ones will change the way it occurs. A weakly insensitive effect is an effect that varies with causes after their force reaches a certain threshold.

Consider the following cases: 


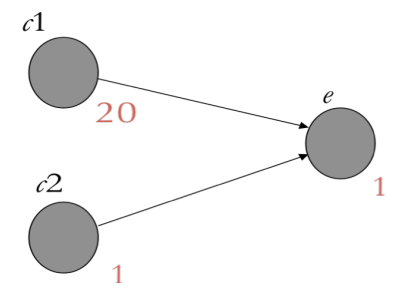

Case 1: Strongly Insensitive

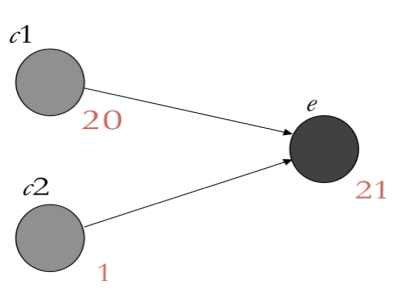

Case 2: Weakly Insensitive

In the first case, $c 1$ fires with a force of $20, c 2$ fires with a force of 1 , and $e$ still fires with a force of 1 . Case 1 has a strongly insensitive effect: even ramping up the force of one of the causes does not change the nature of the effect. This is analogous to a real life case in which the effect is extraordinarily physically rigid. ${ }^{15}$

As a contrast, consider the second case, in which the effect varies when $c 1$ becomes extremely forceful. This is a weakly insensitive effect: it remains rigid before the force of a cause reaches a certain threshold (as when the force of $c 1$ equals 1 or 2), but past a certain threshold, the effect begins to vary with the cause. An example is the following: imagine a window that is rigged to an alarm that is insensitive within the range of four to six rock-throws, but sounds with volume correlative to force below four rockthrows and above six rock-throws. If four, five, or six delinquents throw rocks, the alarm will sound in exactly the same way. But if three or seven delinquents throw rocks, the alarm will sound with lesser or greater volume. In this case, the effect is counterfactually sensitive above or below a certain threshold of force, and insensitive within the threshold.

An effect is counterfactually insensitive (or "modally stable") if it could have occurred differently. Counterfactual insensitivity can be a simple byproduct of modally robust events. For example, if the essence of some window's shattering is just some or other specific window shattering, whenever and however it occurs, then $e$ is counterfactually insensitive across a range of physical differences.

The distinction between physical and counterfactual insensitivity is not steadfast, because the former might be considered a case of the latter: if the nature of an effect does not vary when brought about by differing amounts of physical force, then it is also counterfactually invariant under differing suppositions about the force in operation. A

\footnotetext{
${ }^{15}$ Lest one is skeptical that these exist, consider that a piece of material at the temperature absolute zero is completely impervious to forces and changes in temperature.
} 
counterfactual theorist unimpressed by physical insensitivity might hold that the causal difference between overdetermination and joint causation is entirely captured via counterfactual insensitivity. But not much hinges on the strength of this distinction, as long as we realize that physical insensitivity is the one in play in clear cases of overdetermination. Even if physical insensitivity is a special kind of counterfactual insensitivity, it is physical insensitivity that creates overdetermination cases not vulnerable to JOINT. The more physical insensitivity an event has, the less plausible it is to say that the physical contribution of each cause is necessary for the effect's occurrence.

Inattention to physical insensitivity lies at the heart of the confusion about whether Two Rocks is joint causation or overdetermination. Effects that are counterfactually insensitive but not physically insensitive are vulnerable to JOINT. Two Rocks is such a case: the window shattering occurs whether there are one or two rock-throws, but it shatters differently. By taking Two Rocks to be a case of overdetermination, one stipulates that counterfactual insensitivity is what matters in overdetermination cases. This stipulation is helpful when we are interested in the counterfactual structure of redundant causation, but not helpful when we are interested in event identity. Since physical insensitivity renders an overdetermined effect impervious to change, physical insensitivity plus greater counterfactual sensitivity is what identifies cases of overdetermination that cannot be easily dismissed as joint causation on the basis of modal fragility.

The significance of cases of overdetermination with physically insensitive effects is that they aren't easily eliminable by JOINT. Now, a defender of JOINT might still insist that insensitivity cases are joint causation, as follows:

Tenacious Defender: you classify these newfangled insensitivity cases as overdetermination, and you claim they are actualized. But if we examine them closely enough, we will see that there are still differences in the way the effect occurs. In (Window Alarm), for example, the extra charge running up to the alarm gives it slightly different electrical properties than if it had been singly caused, even if it doesn't necessarily affect the volume of the alarm.

Two responses. First: Tenacious Defender draws a wider spatiotemporal circle around the effect than necessary. Tenacious Defender has misdescribed the case to 
include the intermediate cause: the electrical current. If one examines only the sounding of the alarm and not the events immediately preceding or following it (such as the electricity running through the circuitry), the sounding of the alarm will be exactly the same.

Second: the difference between sensitive effects and insensitive effects is a matter of degree rather than a matter of kind. According to this view, overdetermination and joint causation are on a continuum. On one end, there are strongly insensitive effects such as soundings of alarms encased in nuclear blast-proof titanium, and on the other end, there are highly sensitive effects such as window-shatterings. Taking the distinction to be a continuum does not lessen its importance for understanding why Two Rocks is less vulnerable to JOINT than Window Alarm: physical insensitivity is a property of the latter but not the former. The goal is not so much to have a steadfast distinction between overdetermination and joint causation, but to explain why certain cases are extremely susceptible to JOINT whereas others are not. Even a continuum of sensitivity to insensitivity can undergird this explanation.

\section{Trumping as Overdetermination with Insensitivity}

Schaffer (2000a) introduces trumping preemption as a distinct causal structure from both late preemption and overdetermination. Trumping preemption is a counterexample to counterfactual accounts of causation that attempt to deal with redundant causation by relying on chains of counterfactual dependence in complete causal processes. Here is Schaffer's illustrative case of trumping preemption, which I'll call Magic:

"Imagine that it is a law of magic that the first spell cast on a given day match the enchantment at midnight. Suppose that at noon Merlin casts a spell (the first that day) to turn the prince into a frog, that at 6:00 PM Morgana casts a spell (the only other that day) to turn the prince into a frog, and that at midnight the prince becomes a frog. Clearly, Merlin's spell (the first that day) is a cause of the prince's becoming a frog and Morgana's is not, because the laws say that the first spells are the consequential ones." [Schaffer, 2000]

According to Schaffer, Magic isn't a case of late preemption because both causal 
processes "run to completion," and Magic isn't overdetermination because one cause, Merlin's spell, is intuitively the cause of the enchantment.

Lewis (1999) holds that his "influence" theory of causation accounts for cases of trumping. According to that theory, causation is the ancestral of counterfactual covariation between modally fragile alterations on $c$ and on $e$. In other words: if altering whether, when and how $c$ occurs causes a corresponding alteration in whether, when, and how $e$ occurs, then $c$ is a cause of $e$. Lewis holds that influence handles trumping cases because altering the trumping process, but not the trumped process, produces corresponding changes in the outcome. For example, altering Merlin's spell changes the outcome whereas altering Morgana's spell does not.

With respect to trumping and causation as influence, I will now suggest two related points. First: some cases of trumping are, in fact, overdetermination with insensitive effects. Second, Lewis' influence theory is not well-equipped to handle these cases.

Consider, first, that the enchantment in Magic is underdescribed. Here's what we know: both Merlin's spell and Morgana's spell are complete causal processes individually sufficient to bring about the enchantment in precisely the way that it occurs. ${ }^{16}$ Each reaches all the way to the effect-"hits" the effect, as it were. And we also know that the addition of the extra enchantment doesn't change the way the effect occurs-either way, it will occur in the same way at midnight. A key implicit stipulation of the example is that the enchantment is physically insensitive to the additive force of both spells: removing one spell does not change the time or way that the enchantment occurs, and the presence of both spells does not make the effect occur differently. Given two complete causal processes sufficient to bring about the enchantment, and given an insensitive effect, my analysis classifies Magic as a case of overdetermination. ${ }^{17}$

\footnotetext{
16 It is a complicated and controversial whether both causal processes do, in fact, run to completion in these cases. In Bernstein (2014b) I distinguish between several types of causal completion, and show that trumped and trumping causal processes exhibit no differences in types of causal completion. Since the current discussion is centered around insensitive effects, I do not here address causal completion in detail.

${ }^{17}$ Hitchcock (2011) and Paul and Hall (2013) have recently suggested that some cases of trumping are overdetermination based on ambiguity in what it is to be a "complete" causal process is. I agree with these views. In [AUTHOR], I give an extended analysis of different types of causal completion, and argue that no distinction between types of causal completion renders trumping a distinct form of redundant causation.
} 
To see why the enchantment is an insensitive effect, consider the following contrast case:

(Punk Magic) Imagine that it is a law of magic that the first spell cast on a given day match the enchantment at midnight, unless two spells agree. If two magical spells agree, then the enchantment occurs in a different way. Suppose that at noon Merlin casts a spell (the first that day) to turn the prince into a frog, that at 6:00 PM Morgana casts a spell (the only other that day) to turn the prince into a frog, and that at midnight the prince becomes a punk frog.

Note that we haven't changed the stipulated laws or causes in Schaffer's initial case, but have only added an extra law concerning what happens to the enchantment if the spells agree. The additional law doesn't mandate that the enchantment differs from the content of the first spell if the spells agree (for the prince still turns into a frog), but merely that the enchantment occurs in a different way. We've changed the insensitive effect in Magic to a sensitive effect in Punk Magic, making the latter a case of joint causation. The difference between Magic and Punk Magic is only in the sensitivity of the effect. That pair of cases is structurally similar to the insensitive and sensitive alarm-soundings, in which the effect distinguishes joint causation from overdetermination. The effect is key for the causal diagnosis of each case.

Moreover, a strongly insensitive effect is invariant with respect to alterations on either the trumped or trumping process. Consider that, in Magic, the enchantment occurs at midnight and in a particular manner even if alterations on Merlin's or Morgana's processes occur. Assuming that Merlin's spell occurs before Morgana's spell in every nearby unactualized alteration, then varying either spell individually won't change whether, when, and how the enchantment occurs. For example, if Merlin's spell is cast one second later and an inch to the left, then the enchantment occurs no differently. Similarly with Morgana's spell: if it is cast one second earlier and one inch to the right, the enchantment occurs no differently. Due to the insensitivity of the enchantment, there is no causal asymmetry between Merlin's and Morgana's spells. The general lesson is that causes do not necessarily exhibit Lewisian influence on their effects when those effects are strongly insensitive.

Schaffer's other paradigmatic case of trumping preemption also comes out as overdetermination when fully described. That case, which I'll call Charge, is as follows: 
"Imagine that in a world that could well be our own, the major and the sergeant stand before the corporal, both shout "Charge!" at the same time, and the corporal decides to charge. Orders from higher-ranking soldiers trump those of lower rank." [Schaffer, 2000]

According to Schaffer, both orders run to completion, but the soldiers are only causally responsive to the higher-ranking officer.

Again, the nature of the effect is underdescribed. Fully described, a key stipulation of the case is that how the charge occurs is insensitive to the multiple orders: either order could be removed and the charge would not occur differently. Because both causal processes run to completion, the processes are individually sufficient to bring about the effect, and the effect is insensitive, it is a case of overdetermination. To see why the effect in Charge is insensitive, consider a contrast case:

(Patriotic Charge) The major and the sergeant stand before the corporal, and both shout "Charge!" at the same time. Orders from higher-ranking soldiers trump those of lower rank; however, military law stipulates that if two superiors give an order, the order must be carried out while bearing flags.

Patriotic Charge differs from Charge only in the additional law concerning what happens if both superiors give orders. Patriotic Charge has an effect that is sensitive to the extra cause: it takes both superiors yelling orders to turn the charge into a patriotic one.

One might wonder why insensitivity of the effect marks these cases as overdetermination rather than preemption, given that preempted causes also don't vary counterfactually with the addition or subtraction of a cause. But recall that one typical mark of overdetermination is that both causes are individually sufficient to bring about the effect at precisely the time that it occurs, and in neither early nor late preemption is this case. ${ }^{18}$

\footnotetext{
${ }^{18}$ Another challenge in this strain comes from Yablo's "smart rock," in which Billy's rock is equipped with a satellite guidance system that ensures his rock will shatter the window at precisely the time that Suzy's rock would if Suzy's rock failed to do the job. To this case there are two responses. First: it is not clear that we shouldn't call this case overdetermination based on the individual sufficiency of each cause to bring about the effect in precisely the way that it occurs. Normally actual sufficiency to bring about an effect is the mark of an overdetermining cause, but sometimes a cause's being counterfactually sufficient to bring about the effect suggests overdetermination. Second, insensitivity is the marker of clear cases of overdetermination; that it doesn't categorize outré cases isn't a mark against it. (See [AUTHOR] for an analysis of these cases.)
} 
The defender of trumping-as-preemption has several possible responses against the allegation that trumping is overdetermination. She could hold that what is distinctive about trumping is that both causes are not "causally on par." 19 This means that each cause does not make the same causal contribution to $e .{ }^{20}$ The idea is that Merlin's spell is a cause of the enchantment but Morgana's spell isn't, and the Major's order is a cause of the charge but the Sergeant's order isn't. Due to the causal asymmetries, neither is a case of overdetermination.

But the putative causal asymmetry in cases of trumping is suspicious. Normally what produces causal asymmetry between preemptive redundant causes is that one causal process runs to completion while the other doesn't. But in Magic and Charge, both causal processes are, by stipulation, complete. We are left with an intuition that one process is a cause and the other isn't. But mere intuitive disparity isn't causal disparity. In both cases, either process is sufficient for the effect to occur in exactly the way that it does. No causal process is interrupted. Nothing in the causal structure of the case, as opposed to the laws, produces a causal asymmetry between the two processes.

The defender of trumping-as-preemption could hold that it just is the laws that generate the causal asymmetry. Since the laws stipulate the efficacy of one cause over the other, she holds, the cases are preemption rather than overdetermination.

But this view places undue weight on laws in distinctions between different kinds of redundant causation. In response to this objection, Schaffer gives an extended defense of the supervenience of laws on facts that I will not rehearse here. But we should find the general line of defense unsatisfying. If distinguishing between various species of redundant causation depends on the actual events of the world minus the laws, we should care about causal asymmetries between causal processes rather than laws. These asymmetries are not found in trumping cases. Given the actual "stuff of the world" minus the magical laws, trumping comes out as overdetermination. ${ }^{21}$ Perhaps there is an

\footnotetext{
${ }^{19}$ Schaffer (2000a) holds that overdetermination is characterized by two causes being "causally on par" with respect to an effect.

${ }^{20}$ Schaffer, private communication, and also Schaffer (2003).

${ }^{21}$ The defender of trumping-as-preemption could just hold that what she calls trumping, I call overdetermination. But the disagreement is not merely verbal: to deny causal asymmetry between the two causes is to engage in a metaphysically substantive debate over the existence and source of the causal asymmetry.
} 
alternative conception of redundant causation that prioritizes laws over actual causal processes, but this is not the type of redundant causation upon which our distinctions between species of redundant causation should be based.

One might further defend the use of laws by holding that nomic asymmteries just are causal asymmetries, perhaps irreducible ones. ${ }^{22}$ Such a conception links laws and causation, and represents the commonly accepted close relationship between causation and lawful necessity. But note that the law at work in a case of trumping is an unconvincing stand-in for a natural law or laws. No natural law decides, ex post facto, which physical process is the cause of an outcome. Laws in our world antecedently determine when something will be a cause of something else, but don't primitively prioritize one complete causal process over another. Thus even if one is friendly to the Schafferian interpretation of the example, it is uninformative with respect to causation at our world.

Further, note that even if one is friendly towards law-generated asymmetry, this consideration does not undermine the more general point that insensitivity is the best way to understand cases of trumping - including their close resemblance to overdetermination. With an insensitive effect, both causes are sufficient to bring about the outcome in exactly the way that it occurs. Whether one collapses or maintains the trumping/ overdetermination distinction, insensitivity of the outcome explains why it isn't easily reducible to a case of joint causation.

\section{Conclusion}

I have isolated a special sort of overdetermination-overdetermination with insensitive effects - and shown how it differs from joint causation. This sort of overdetermination isn't vulnerable to the objection that all overdetermination is just joint causation underdescribed, and teaches us why some cases tempt this conclusion while others don't. Insensitivity cases also provide an alternative analysis of putative cases of trumping preemption. One advantage of the analysis is that we no longer have an extra category of redundant causation.

22 Thanks to a referee for this objection. 


\section{References}

Bernstein, S. (2014) “A Closer Look at Trumping”. Acta Analytica. -------- (MS) "Overdetermination Underdetermined".

Bunzl, M. (1979) “Causal Overdetermination”, Journal of Philosophy 76, 134-150.

Davidson, D. (2001) “The Individual of Events", Essays on Actions and Events (Oxford: Oxford University Press 2001) 163-80.

Dowe, P. (2000) Physical Causation. New York: Cambridge University Press.

Hall, N. and Paul, L. (2013) "Causation and the Counterexamples: a User's Guide." Oxford: Oxford University Press.

Hitchcock, C. (2011) “Trumping and Contrastive Causation.” Synthese 181:227-240.

Kim, J., 1976, "Events as Property Exemplifications", in M. Brand and D. Walton (eds.), Action Theory, Dordrecht: Reidel, pp. 159-77.

Merricks, T. (2007) Objects and Persons Oxford: Oxford University Press.

Paul, L. (2007) "Constitutive Overdetermination" in Topics in Contemporary Philosophy, Vol IV: Causation and Explanation.

Schaffer, J. (2000a) “Trumping Preemption”, Journal of Philosophy 97 (4):165-181. --------- (2003) “Overdetermining Causes” Philosophical Studies 114: 23-45.

Sider, T. (2003) "What's so Bad about Overdetermination?" Philosophy and Phenomenological Research 67: 719-726. 
